\title{
Risques de limitation en lysine et méthionine des «rations vaches laitières» : étude à partir de 55 rations
}

\author{
N Jouan, F Laurent, O Colin
}

\begin{abstract}
INRA-ENSAIA, laboratoire de sciences animales, 2, avenue de la Forêt-de-Haye, $B P$ 172, 54505 Vandouvre-lès-Nancy cedex, France
\end{abstract}

Cinquante-cinq rations à base d'ensilage de maïs complémenté majoritairement avec du maìs ou du soja et/ou de la luzerne, provenant d'essais de supplémentation en lysine (Lys) et/ou méthionine (Met) et couvrant les besoins des animaux, ont permis de définir 3 groupes en fonction de la réponse du taux protéique (TP) à l'apport de Lys ou de Met. Le jer groupe comprend les rations répondant à l'apport de Lys (15 rations) et le $2^{8}$ celles répondant à l'apport de Met (11 rations) $(+0,7 \mathrm{~g} / \mathrm{kg}$ de TP au moins par rapport au témoin ou à la dose immédiatement inférieure, la production laitière ne devant pas diminuer de plus de $1 \mathrm{~kg}$ ) ; le $3^{\theta}$ groupe rassemble les rations ne répondant pas à l'apport de Lys et/ou de Met (29). Une analyse factorielle discriminante (AFD, STATITCF 5.0) est réalisée en utilisant la production laitière $4 \%$, les taux butyreux et protéique, les quantités de protéines produites, les teneurs en matières azotées totales et en matières grasses, les apports estimés en PDIA, PDIMN et $P D I M E$ de la ration, les pourcentages de Lys et de Met digestibles dans les PDI limitantes (\% Lys ou Met dans l'aliment x PDIA + \% Lys ou Met dans les bactéries [Le Hénaff, 1991] x PDIM limitantes) et le rapport Lys/Met digestibles. L'AFD réalisée est ensuite utilisée pour classer 76 autres rations, à base d'ensilage de maïs, provenant d'essais de supplémentation pour lesquels l'origine de la supplémentation (Lys ou Met) n'a pu être déterminée ou ne provenant pas d'essais de supplémentation, dans l'un des 3 groupes préalablement définis.
Les variables les plus discriminantes pour classer les 55 rations sont le pourcentage de Lys digestible dans les PDI totales et le rapport Lys/Met digestible. Les parts moyennes de Lys et Met digestibles dans les PDI totales sont, pour le groupe des rations répondant à la supplémentation en Lys, respectivement de 4,3 et 2,5\% pour le groupe des rations répondant à la supplémentation en Met, de 6,5 et 2,1\% et pour le groupe des rations ne répondant pas à la supplémentation de 5,8 et 2,2\%. Les rapports Lys/Met digestibles sont respectivement pour les 3 groupes de $1,7,3,1$ et 2,7 . Des 76 rations utilisées comme individus supplémentaires, 12 sont classées dans le groupe 1, 42 dans le groupe 2 et 22 dans le groupe 3. Les parts de Lys et Met digestibles dans les PDI totales sont respectivement de 5,2 et 2,15 pour les rations du groupe $1 ; 6,2$ et 2,08 pour les rations du groupe $2 ; 5,8$ et 2,06 pour les rations du groupe 3 .

Les risques de limitation de la quantité de protéines du lait produites sont faibles pour un pourcentage de Lys digestible dans les PDI totales supérieur à $6,5 \%$ et de Met supérieur à 2,5\%. Pour les rations du $3^{\theta}$ groupe, l'un des 2 acides aminés ou les 2 peuvent être limitants. L'utilisation du modèle permet de prévoir les risques de limitation en Met ou Lys à partir des caractéristiques nutritionnelles des rations et des performances de production des vaches.

Le Hénaff $L$ (1991) Thèse de doctorat de l'université de Rennes I 